\title{
Thermoelastic and Pseudoelastic Characterization of Shape Memory Alloys
}

\author{
Osman Adiguzel ${ }^{*}$ \\ Department of Physics, Firat University, Elazig, Turkey. \\ * Corresponding author. email: oadiguzel@firat.edu.tr \\ Manuscript submitted April 10, 2017; accepted August 18, 2017. \\ doi: 10.17706/ijmse.2017.5.3.95-101
}

\begin{abstract}
Shape-memory effect is based on martensitic transformation, which is a solid state phase transformation and govern the remarkable changes in internal crystalline structure of materials. Shape memory alloys can exhibit another property called pseudoelasticity (PE), which is performed in only mechanical manner in the parent austenite phase region. Shape Memory Effect (SME) is performed thermally in a temperature interval and this behaviour is called thermoelasticity. Pseudoelasticity is performed by stressing the material in the parent phase region, and shape recovery is performed simultaneously upon releasing the applied stress. PE is the result of stress-induced martensitic transformation. Martensitic transformations occur with cooperative movement of atoms by means of lattice invariant shear which occurs in two opposite directions on close packed planes of parent austenite phase, and possible 24 martensite variants occur. By this way the twinned martensite occurs on cooling, and the twinned structure turn into the detwinned martensite by deformation in the low temperature product phase condition. Copper based alloys exhibit this property in metastable beta-phase region, which has bccbased structures at high temperature parent phase field, and these structures martensiticaly turn into layered complex structures with lattice twinning on cooling.
\end{abstract}

Keywords: Shape memory effect, martensitic transformation, thermoelasticity, pseudoelasticity, twinning and detwinning.

\section{Introduction}

Phase transformations are important factor in the processing of materials, like strengthening the steel and shape changes with variation of temperature. Shape memory alloys take place in a class of functional materials by exhibiting a peculiar property called shape memory effect. This property is characterized by the recoverability of desired shape on the material at different temperatures. Shape memory effect is performed in only thermal manner on heating and cooling after deformation at low temperature martensitic condition, and this behavior can be called thermoelasticity. Shape memory effect is linked with martensitic transformation, and comprises a reversible transition from product martensite to parent austenitic phase. Martensitic transformations are first order lattice-distorting phase transformations and occur with the cooperative movement of atoms by means of lattice invariant shears in the materials on cooling from high temperature parent phase region. Martensitic transformation is evaluated by the structural changes in microscopic scale. Shape memory effect is based on martensitic transformation, and microstructural mechanisms are responsible for shape memory behaviour. In particular, the twinning and detwinning processes are essential as well as martensitic transformation in reversible shape memory effect [1], [2]. 
Thermal induced martensite occurs by means of a shear-like mechanism as multivariant martensite in selfaccommodating manner and consists of lattice twins. Also, this martensite is called twinned martensite or multivariant martensite. Martensitic transformations occur with cooperative movement of atoms by means of lattice invariant shears on a $\{110\}$ - type plane of austenite matrix which is basal plane of martensite. The lattice invariant shears occurs, in two opposite directions, $<110>$-type directions on the $\{110\}$-type basal planes. The $\{110\}$-plane family has 6 certain lattice planes; $\{110\},\left\{\begin{array}{lll}1-1 & 0\end{array}\right\},\{101\},\left\{\begin{array}{ll}1 & 0-1\end{array}\right\},\{011\},\left\{\begin{array}{ll}0 & 1-1\}\end{array}\right\}$. This kind of shear can be called as $\{110\}<110>$ - type mode and possible 24 martensite variants occur. Shape memory alloys can be deformed plastically in low temperature martensitic condition, and recover the original shape on heating over the austenite finish temperature. The material cycles between the deformed and original shapes on cooling and heating in reversible shape memory case. By applying external stress, martensite variants are forced to reorient into a single variant leading inelastic strains, and deformation of shape memory alloys in martensitic state proceeds through a martensite variant reorientation or detwinning of twins [1], [2]. The twinning occurs with internal stresses, while detwinning occurs with the external stresses. The basic mechanism phase transition and shape memory effect in crystallographic level is schematically illustrated in Fig. 1 [2]. The deformed material recovers the original shape in bulk level, and crystal structure turns into the parent phase structure on first heating.

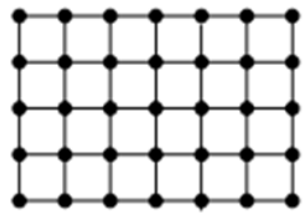

( a )

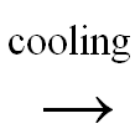

deform

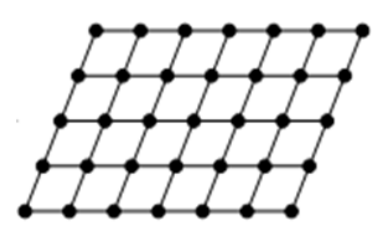

( c )

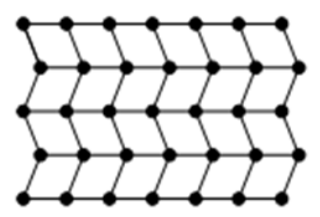

(b)

Fig. 1. Schematic illustration of the mechanism of the shape-memory effect: (a) atomic configuration on $\{110\}$-type planes of parent austenite phase, (b) twinned martensite phase occurring thermally on cooling, (c) detwinned martensite occurring with deformation [2].

The parent phase structure returns to the multivariant martensite structures in irreversible shape memory effect on cooling below the martensite finish temperature; in contrast, the material returns to the detwinned martensite structure in reversible shape memory case. Shape memory alloys exhibit another property, superelasticity (SE), which is performed by mechanical stress. Shape memory alloys can be deformed just over austenite finish temperature, and recover the original shape on releasing the stress in superelastic manner. Deformation at different temperature exhibits different behaviour beyond shape memory effect and superelasticity.

Shape memory effect is performed in a temperature interval depending on the forward (austenite $\rightarrow$ martensite) and reverse (martensite $\rightarrow$ austenite) transformation, on cooling and heating, respectively. Superelasticity is performed in the parent austenite phase region, just over Austenite finish temperature. Superelastic materials are deformed in the parent phase region and, shape recovery is carried out instantly and simultaneously upon releasing the applied stress. This property exhibits rubber like behaviour or classical elastic material behaviour. Stress-strain behaviour is different in two cases, shape memory effect and super-elasticity. Deformation is performed plastically in product martensitic condition in shape memory case. Meanwhile, the material is deformed in parent phase region in superelasticity, and the material recover 
the original shaper after releasing the external stress. Superelasticity proceeds with only mechanical treatment. This behaviour is also result of martensitic transformation, stress induced martensitic transformation, which is induced by applying external stress only in mechanical manner. With this stress, parent austenite phase structures turn into the fully detwinned martensite, and very large strain is observed; and a complete shape recovery is observed upon unloading, therefore, the material behavior resembles elasticity. This strain is maximum 8\% in NiTi alloys [2]. The loading and releasing paths demonstrating the shape memory and superelastic effects and stress-strain diagram is shown in Fig. 2 [3].

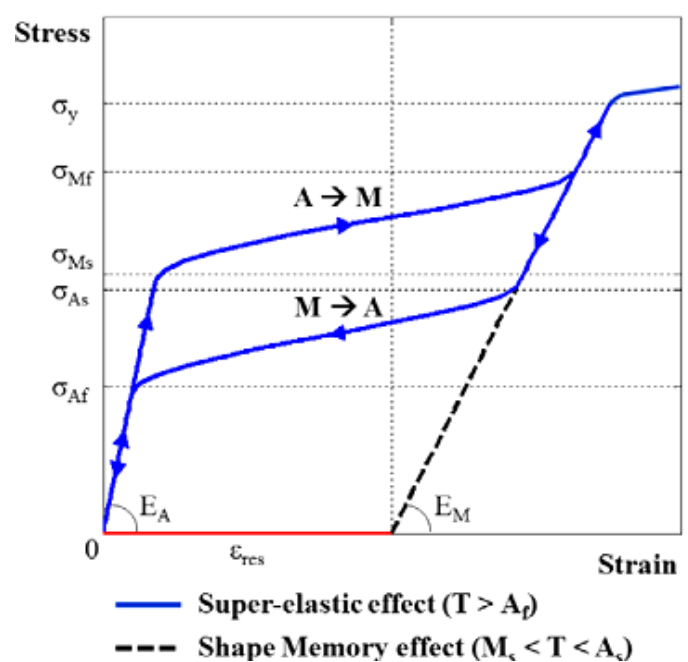

Fig. 2. Stress-strain diagram of shape memory effect and superelasticity [3].

Copper based alloys exhibit this property in metastable $\beta$-phase region, which has $\mathrm{B} 2$ or $\mathrm{DO}_{3}$-type ordered lattice at high temperature, and these structures martensiticaly turn into layered complex structures with lattice twinning process, on cooling from high temperature austenitic phase region. Martensitic transformations occur mainly in two steps in copper based ternary alloys.
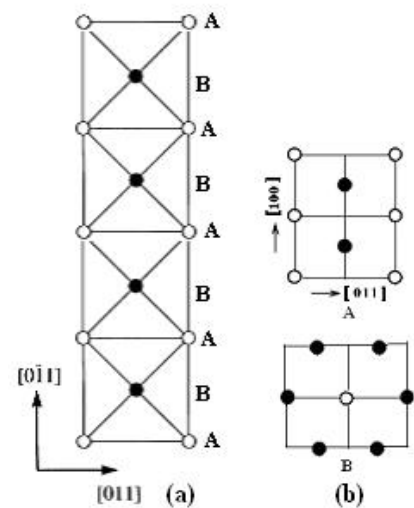

(b)

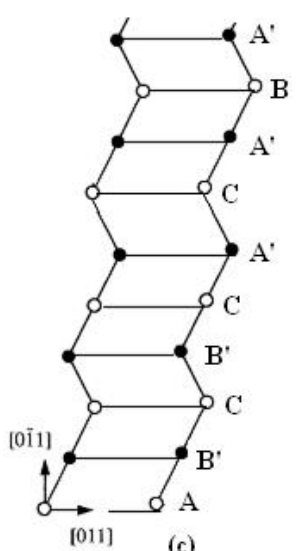

(c)

Fig. 3. (a) Stacking of (110) $\beta$ planes viewed from (001) $\beta$ direction,(b) Atomic configuration on first and second layers of (110) $\beta$ plane in D03 - type structures, (c) inhomogeneous shear and formation of layered structures, stacking sequences of half 18R or M18R unit cell in direction $\mathrm{z}$.

First one is Bain distortion, and second one is lattice invariant shear. Bain distortion consists of an expansion of $26 \%$ parallel to the $<001>$-type axes, and compression of $11 \%$ parallel to the $<110>$ and $<1 \overline{1} 0>$-type directions [4]. Lattice invariant shears occur with cooperative movement of atoms less than interatomic distances on $\{110\}$-type close packet plans of austenite matrix. The lattice invariant shears occur, 
in two opposite directions, $<110>$-type directions on the $\{110\}$-type basal planes and this type of shear can be called as $\{110\}<110>$ - type mode and has 24 variants in self-accommodating manner [5]-[8]. These lattice invariant shears are not uniform in copper alloys and give rise to the formation of unusual complex layered structures called long period layered structures such as 3R, 9R or 18R depending on the stacking sequences on the close-packed planes of the ordered lattice. Formation of these layered structures is schematically illustrated in Fig. 3. All of these martensite phases are long-period stacking ordered structures that is the underlying lattice is formed by stacks of close-packed planes. In case the parent phase has a B2-type superlattice, the stacking sequence is $\mathrm{ABCBCACAB(9R)}$ [5]-[7].

\section{Experimental Details}

In the present paper, two copper based ternary shape memory alloys were selected for investigation; $\mathrm{Cu}-$ 26.1\% $\mathrm{Zn} 4 \% \mathrm{Al}$ and $\mathrm{Cu}-11 \% \mathrm{Al}-6 \% \mathrm{Mn}$ (in weight). The martensitic transformation temperatures of these alloys are over the room temperature and both alloys are entirely martensitic at room temperature. Specimens obtained from these alloys were solution treated for homogenization in the $\beta$-phase field (15 minutes at $830^{\circ} \mathrm{C}$ for $\mathrm{CuZnAl}$ alloy and 20 minutes at $700^{\circ} \mathrm{C}$ for CuAlMn alloy), then quenched in iced-brine to retain the $\beta$-phase and aged at room temperature after quenching ( both alloys).

Powder specimens for X-ray examination were prepared by filling the alloys. Specimens for TEM examination were also prepared from $3 \mathrm{~mm}$ diameter discs and thinned down mechanically to $0.3 \mathrm{~mm}$ thickness. These specimens were heated in evacuated quartz tubes in the $\beta$-phase field (15 minutes at $830^{\circ} \mathrm{C}$ for CuZnAl and 20 minutes at $700^{\circ} \mathrm{C}$ for CuAlMn) for homogenization and quenched in iced-brine. These specimens were also given different post-quench heat treatments and aged at room temperature. TEM and X-ray diffraction studies carried out on these specimens. TEM specimens were examined in a JEOL 200CX electron microscope, and X-ray diffraction profiles were taken from the quenched specimens using $\mathrm{Cu}-\mathrm{K}_{\alpha}$ radiation with wavelength $1.5418 \AA$.

\section{Results and Discussion}

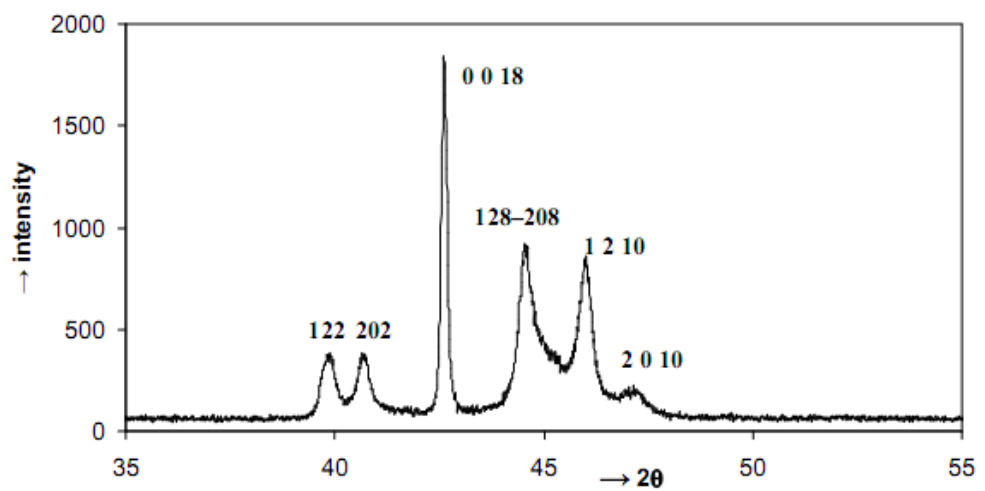

Fig. 4. An x-ray diffractogram taken from the long term aged CuAlMn alloy sample.

X-ray powder diffractograms were taken from CuZnAl and CuAlMn samples. An x-ray powder diffractogram taken from the long term aged CuAlMn alloy sample is shown in Fig. 4. This diffractogram has been indexed on the monoclinic M18R basis. X-ray diffraction profile of CuZnAl alloy also exhibit similar configuration. Two electron diffraction patterns taken from $\mathrm{CuZnAl}$ alloy sample are also shown in Fig. 5, respectively. X-ray powder diffractograms and electron diffraction patterns reveal that these alloys exhibit superlattice reflections. X-ray diffractograms and electron diffraction patterns reveal that both alloy have the ordered structure in martensitic condition, and exhibit superlattice reflections. A series of X-ray powder 
diffractograms and electron diffraction patterns were taken from both CuZnAl and CuAlMn alloy samples in a large time interval and compared with each other. It has been observed that electron diffraction patterns exhibit similar characteristics, but some changes occur at the peak locations and intensities on the x-ray diffractograms with aging duration. These changes occur as rearrangement or redistribution of atoms in the material, and attribute to new transitions in diffusive manner [4], [7], [11]. The ordered structure or super lattice structure is essential for the shape memory quality of the material. In the shape memory alloys, homogenization and releasing the external effect is obtained by ageing at $\beta$-phase field for adequate duration.
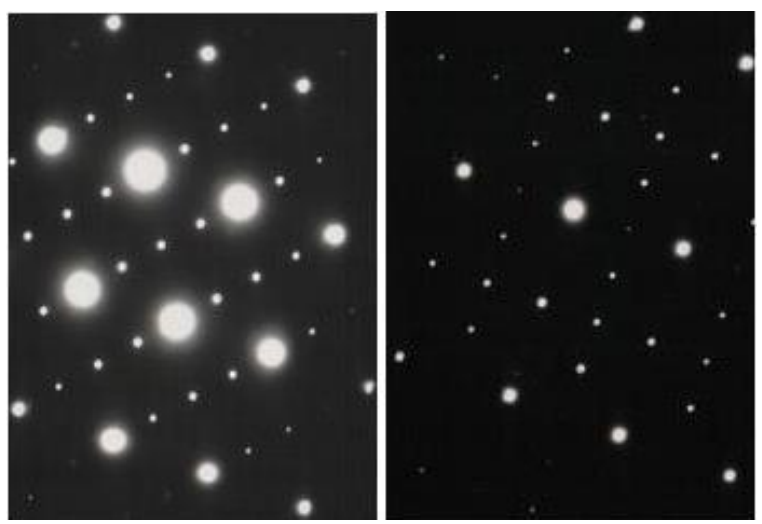

Fig. 5. Two electron diffraction patterns taken from the CuZnAl and CuAlMn alloy samples.

On the other hand, post-quench ageing and service processes in devices affect the shape memory quality, and give rise shape memory losses. These kinds of results lead to the martensite stabilization in the reordering or disordering manner. In order to make the material satisfactorily ordered and to delay the martensite stabilization, copper-based shape memory alloys are usually treated by step-quenching after homogenization. Metastable phases of copper-based shape memory alloys are very sensitive to the ageing effects, and any heat treatment can change the relative stability of both martensite and parent phases [11], [12]. Martensite stabilization is closely related to the disordering in martensitic state.

Structural ordering is one of the important factors for the formation of martensite, while atom sizes have important effect on the formation of ordered structures [9], [10], [12]. Although martensitic transformation has displacive character, martensite stabilization is a diffusion controlled phenomena, and this result leads to redistribution of atoms on the lattices sites. Stabilization is important factor and causes to memory losses, and changes in main characteristics of the material; such as, transformation temperatures, and diffracted angles and peak intensities.

Martensitic transformation in copper-based $\beta$-phase alloys is based on one of the $\{110\}_{\beta}$ planes of parent phase called basal plane for martensite. The (110) basal plane which has a rectangular shape in parent phase is subjected to hexagonal distortion and undergoes a hexagon.

The powder specimens were aged at room temperature after quenching process, and many x-ray diffractograms have been taken from both of the alloy samples in a large time interval. Although all of the diffractograms exhibit similar characteristics, some changes have been observed at diffraction angles and intensities of diffraction peaks on the diffractograms with aging duration. These changes are attributed to new transitions which have diffusive character. It means that some neighbour atoms change locations. In particular, some of the neighbour peak pairs have moved toward each other. It is interesting that miller indices of these plane pairs provide a special relation:

$$
\left(h_{1}^{2}-h_{2}^{2}\right) / 3=\left(k_{2}^{2}-k_{1}^{2}\right) / n
$$


Where $\mathrm{n}=4$ for $18 \mathrm{R}$ martensite [4]. These plane pairs can be listed as follow; (122)-(202), (128)-(208), (1 2 10) - (2 0 10), (040)- (320). This result can be attributed to a relation between interplane distances of these plane pairs and rearrangement of atoms on the basal plane. In these changes, atom sizes play important role. The different sizes of atomic sites lead to a distortion of the close-packed plane from an exact hexagon and thus a more close-packed layered structure may be expected. In the disordered case, lattice sites are occupied randomly by the atoms, and atom sizes can be taken nearly equal, and martensite basal plane becomes an ideal hexagon, whereas, the lattice sites are occupied regularly by different atoms which have different sizes.

\section{Conclusions}

It can be concluded from the above results that the copper-based shape memory alloys are very sensitive to the ageing treatments. The diffraction angles and intensities of x-ray diffraction peaks gradually change with the ageing duration in martensitic condition. In particular, some successive peak pairs come close each other. These changes lead to the martensite stabilization in the redistribution or disordering manner, and stabilization proceeds by a diffusion-controlled process. The martensite stabilization is a diffusion controlled phenomena and leads to redistribution of atoms on the lattices sites, although martensitic transformation has displacive character. The basal plane of martensite turns into a hexagon by means of Bain distortion with martensite formation on which atom sizes have important effect. In case the atoms occupying the lattice sites have the same size, the basal plane of martensite becomes regular hexagon; otherwise the deviations occur from the hexagon arrangement of the atoms. The above mentioned peaks come close each other in the disordered case, and occur separately in the ordered case. The changes in the diffraction angles of the selected plane pairs can be a measure of the ordering degree in martensite.

\section{References}

[1] Ma, J., Karaman, I., \& Noebe, R. D. (2010). High temperature shape memory alloys. International Materials Reviews, 55(5), 257-315.

[2] Sun, L., Huang, W. M., Ding, Z., Zhao, Y., Wang, C. C., Purnawali, H., et al (2012). Stimulus-responsive shape memory materials: A review. Materials and Design, 33, 577-640.

[3] Barbarino, S., Flores, E. I. S., Ajaj, R. M., Dayyani, I., \& Friswell, M. I. (2014). A review on shape memory alloys with applications to morphing aircraft. Smart Mater. Struct., 23, 1-19.

[4] Adiguzel, 0. (2013). Phase transitions and microstructural processes in shape memory alloys. Materials Science Forum, 762, 483-486.

[5] Zhu, J. J., \& Liew, K. M. (2003). Description of deformation in shape memory alloys from $\mathrm{DO}_{3}$ austenite to 18R martensite by group theory. Acta Materialia, 51(9), 2443-2456.

[6] Sutou, Y., Omori, T., Yamauchi, K., Ono, N., Kainuma, R., \& Ishid, K. (2005). Effect of grain size and texture on pseudoelasticity in Cu-Al-Mn-based shape memory wire. Acta Materialia, 53(15), 4121-4133.

[7] Adiguzel, 0. (2012). Martensitic transformation and microstructural characteristics in copper based shape memory alloys. Key Engineering Materials, 510-511, 105-110.

[8] Casati, R., Vedani, M., \& Tuissi, A. (2014). Thermal cycling of stress induced martensite for high performance shape memory effect. Scripta Materialia, 80, 13-16.

[9] Guo, Y. F., Wang, Y. S., Zhao, D. L., \& Wu, W. P. (2007). Mechanisms of martensitic phase transformations in body-centered cubic structural metals and alloys: Molecular dynamics simulations. Acta Materialia, 55(19), 6634-6641.

[10] Aydogdu, A., Aydogdu, Y., \& Adiguzel, O. (2004). Long-term ageing behaviour of martensite in shape memory Cu-Al-Ni alloys. Journal of Materials Processing Technology, 153-154, 164-169. 
[11] Li, Z., Gong, S., \& Wang, M. P. (2008). Macroscopic shape change of Cu13Zn15Al shape memory alloy on successive heating. Journal of Alloys and Compounds, 452(2), 307-311.

[12] Bubani, F. de C., Sade, M., \& Lovey, F. (2012). Improvements in the mechanical properties of the 18R $\leftrightarrow$ 6R high hysteresis martensitic transformation by nanoprecipitates in CuZnAl alloys. Materials Science and Engineering A, 543, 88-95.

Prof. Osman Adiguzel is from Firat University, Turkey. His research areas are Martensitic phase transformations and shape memory effect and applications to copper-based shape memory alloys, molecular dynamics simulations, alloy modeling, electron microscopy, x-ray diffraction and crystallography, differential scanning calorimetry (DSC). 